\title{
Unusual presentation of scrub typhus in pregnancy with postpartum dilated cardiomyopathy: a case report
}

\author{
Ashita Aggarwal*, Madhu Shweta Sharma
}

Department of Obstetrics and Gynecology, Sikkim Manipal Institute of Medical Sciences Gangtok, Sikkim, India

Received: 05 January 2020

Accepted: 05 February 2020

*Correspondence:

Dr. Ashita Aggarwal,

E-mail: ashitaa.agg@gmail.com

Copyright: (C) the author(s), publisher and licensee Medip Academy. This is an open-access article distributed under the terms of the Creative Commons Attribution Non-Commercial License, which permits unrestricted non-commercial use, distribution, and reproduction in any medium, provided the original work is properly cited.

\begin{abstract}
Though scrub typhus is one of the uncommon causes of fever in peripartum period it must be kept in differential diagnosis of any febrile illness particularly when the signs and symptoms points towards rickettsial etiology. WeilFelix test can be used for diagnosis. Uncommon complications such as cardiomyopathy must be suspected in cases who present with undue dyspnea and signs of congestive heart failure. Patients with cardiomyopathy needs appropriate management in intensive care units. ECG and 2D echocardiography (2D echo) needs to be done in these patients which may show arrhythmias and ventricular hypokinesia and decreased ejection fraction respectively. A 21 years aged first gravida female at 34 weeks of gestational age was admitted with complaints of high-grade fever since 4-5 days with no previous antenatal visits. A diagnosis of rickettsial fever was suspected on the basis of presenting complaints and clinical examination. Weil-Felix test turned out to be positive with OX19 titre of 1:160. She underwent LSCS in view of fetal distress and delivered a healthy male child. In immediate postpartum period she developed respiratory distress. Her 2D echo showed features suggestive of dilated cardiomyopathy. She was managed in medical intensive care unit and was eventually discharged. Though uncommon rickettsial diseases should be kept in the differential diagnosis of any febrile illness particularly when presenting with characteristic rash. Serious complications such as cardiomyopathy must be diagnosed early and treated appropriately in these patients to prevent mortality.
\end{abstract}

Keywords: Cardiomyopathy, Postpartum period, Scrub typhus, 2D echocardiography

\section{INTRODUCTION}

Scrub typhus usually present as an acute febrile illness and is caused by Orientia tsutsugamushi. This disease is endemic in east Asia and pacific islands. Fever in scrub typhus is usually accompanied by headache, rigors and lymphadenopathy. The other clinical features may include splenomegaly, retro-orbital pain, cough and respiratory symptoms. Majority of the patients usually recover, and the disease usually dramatically respond to doxycycline. Rarely serious complications such as shock, renal failure, disseminated intravascular coagulopathy and cardiomyopathy may occur. Unrecognized cardiomyopathy in cases of scrub typhus may have serious consequences such as cardiogenic shock, arrythmias and even cardiac arrest. Cardiomyopathy remains one of the important causes of mortality in cases of scrub typhus. ${ }^{1}$

Scrub typhus in pregnancy is associated with poor fetal outcome. Peri or postpartum, cardiomyopathy is a rare, life threatening heart disease of unclear origin and is characterized by heart failure of sudden onset between the final weeks of pregnancy and 6 months after delivery. Peripartum cardiomyopathy defined as "idiopathic cardiomyopathy presenting with heart failure secondary 
to left ventricular systolic dysfunction towards the end of pregnancy or within 5 months following delivery, where no other cause of heart failure is found". Scrub typhus is one of the important causes of peripartum cardiomyopathy particularly in Asian countries including India. Since scrub typhus also present as febrile illness it is one of the uncommon still an important differential diagnosis of febrile illness in peripartum period. ${ }^{2}$

Cardiomyopathy during peripartum period is poorly understood and carry a wide range of differential diagnoses. Irrespective of the etiology it is characterized by a catastrophic progress which eventually may culminate into serious consequences such as heart failure or even cardiac arrest. Though peripartum cardiomyopathy presents clinically similar to dilated cardiomyopathy it is unique in the sense that it does have a rapidly progressive deterioration of cardiac function as compared to dilated cardiomyopathy. Though with proper supportive care majority of patients with peripartum cardiomyopathy recover there are many patients who have variable amount of residual left ventricular dysfunction. ${ }^{3}$

Since peripartum cardiomyopathy is uncommon a high index of suspicion is needed. Any delay in the diagnosis and treatment may rapidly prove fatal. It must be suspected in any peripartum woman who complains of dyspnea, nocturia, palpitation or undue exhaustion. The signs which may point towards the possibility of cardiomyopathy may include pedal edema, ECG abnormalities, systolic murmur on auscultation or acute onset focal neurological deficit due to infarcts secondary to cerebral emboli. ${ }^{4}$

The incidence of peripartum cardiomyopathy is variable and reported to range between 1:3500 to 1:299. The huge difference in incidence is due to the factors such as race and geographical variations. The common causes of peripartum cardiomyopathy include viral myocarditis, nutritional deficiencies, autoimmune, hormonal insults, and rarely underlying genetics amongst others. The viral causes are reported to be more common as compared to other etiologies and most common viral etiologies includes echovirus, parvovirus B19, HHV 6, Epstein bar virus and Cytomegalovirus. Myocarditis caused by scrub typhus is rarely reported in peripartum period. ${ }^{5}$

This case here reported peripartum dilated cardiomyopathy caused by scrub typhus during immediate postpartum periods. This case report emphasizes the importance of knowing this differential diagnosis of cardiomyopathy in peripartum period.

\section{CASE REPORT}

A 21 years aged first gravida female at 34 weeks of gestational age was admitted with complaints of highgrade fever since 4-5 days with no previous antenatal visits. Fever was associated with body ache and there were no diurnal variations. There was also history of dyspnea and fatigue since 2 days. Family history didn't have any significant cardiac illness in any other family member. Past history was non-contributory. On examination patient was febrile and there was presence of macular rash on trunk. There was no significant lymphadenopathy. On careful examination a popular lesion with central necrosis was found on back of neck. A complete blood count was done which showed lymphocytosis. In view of characteristic lesion on back of neck, macular rash on trunk and presence of lymphocytosis a provisional diagnosis of scrub typhus was made and Weil-Felix test was done. Weil-Felix test turned out to be positive with OX19 titer of 1:160. The diagnosis of scrub typhus was made and patient was put on azithromycin. On day 2 of admission there was fetal distress as seen by fetal bradyarrhythmia's. In view of fetal distress an emergency lower segment cesarean section (LSCS) was done. She delivered a healthy male child weighing $2.25 \mathrm{~kg}$. Baby cried immediately after birth and no resuscitation was required.

In postpartum period ( 2 hours after surgery) patient developed respiratory distress in the form of tachypnea, nasal flaring and irregular respiration. On auscultation bilateral basal crept were present. On examination a persistent tachycardia and pedal edema was also noted. A medicine consultation was sought for tachycardia and respiratory distress and on the advice of physician chest $\mathrm{X}$-ray and urgent 2D echo was done. On X-ray there was a cardiomegaly (suggested by increased cardiothoracic ratio) and pulmonary edema (suggested by air space opacification and perihilar haze). 2D echo showed dilated cardiomyopathy with severe left ventricular systolic dysfunction along with hypokinesia. The ejection fraction was found to be $41 \%$.

In view of dilated cardiomyopathy with reduced ejection fraction patient was shifted to medicine intensive care unit and was started on diuretics (Torsemide), levocarnitine, beta blockers (carvedilol) and ACE inhibitors (Enalapril). Oxygen inhalation was also started. Patient started improving on these medications and her pedal edema started reducing. The tachypnea and tachycardia were also settled on D2 of admission on Medicine intensive care unit. Patient started maintaining saturation without oxygen on D3 of MICU admission and oxygen inhalation was stopped. In view of improved general condition and absence of pedal edema, tachycardia or tachypnea patient was shifted back to PNC ward and breastfeeding was started.

Patient was discharged on day 10 of admission. At the time of discharge patient was symptomatically better with no dyspnea. Her heart rate was $82 / \mathrm{min}$ and pedal edema was absent. A repeat echo was done before discharge which showed left ventricular ejection fraction to be $55 \%$. At the time of discharge patient was counselled about chances of recurrence of peripartum cardiomyopathy in future pregnancies and advised 
insertion of IUCD to which she agreed and hence before discharge an intrauterine contraceptive device was inserted. Patient was advised to continue medication and avoid undue exertion and was called for follow up in OPD after 1 week.

\section{DISCUSSION}

The etiology of cardiomyopathy is varied and may consist of ischemia (ischemic cardiomyopathy), hypertension (hypertensive cardiomyopathy), infiltrative (sarcoidosis), infective (HIV, CMV, toxoplasmosis, ricketsial), drug induced (Doxorubicin, amphetamines), diabetes and hyperthyroidism. Like any other cardiovascular disorder, it is more common in males as compared to females.

Amongst the infective causes viral infections are most common cause of myocarditis. Ricketsial diseases causing myocarditis is a rare occurrence. Scrub typhus is one of the uncommon causes of cardiomyopathy and its occurrence during pregnancy is rarely reported. Cardiomyopathy during pregnancy puts an additional burden on already compromised cardiovascular system and sometimes it becomes difficult to identify cardiomyopathy during pregnancy or in immediate postpartum period since the symptoms such as fatigue and dyspnea is erroneously attributed to pregnancy. Scrub typhus usually present with fever rash and lymphadenopathy. Serious complications such as disseminated intravascular coagulopathy, renal failure, Encephalopathy and cardiomyopathy can be seen in some patients particularly in cases where treatment is delayed. Rapsang AG et al found that age more than 60 years, leukocytosis $(>10,000 / \mathrm{mm})$ and hypoalbuminemia $(\leq 3.0$ $\mathrm{g} / \mathrm{dL}$ ) were the predictors of complications in patients with scrub typhus. ${ }^{6}$

The cardiomyopathy and myocarditis due to scrub typhus has been reported rarely. When present it usually manifest clinically by pedal edema, raised jugular venous pressure and dyspnea. On X-ray signs of pulmonary edema may be present along with increased cardiothoracic ration s/o cardiomegaly. 2D echo may show hypokinetic ventricles along with decreased ejection fraction. Hypokinesia of left ventricle is the most sensitive finding on 2D echo in cases of cardiomyopathy. Neiman MS et al found that hypokinesia was the only finding on echo in cases of mild cases of myocarditis. ${ }^{7}$

This case brings forward 2 important points. First is to identify the incidence of cardiomyopathy in peripartum period. It must be emphasized that clinical features such as pedal edema and dyspnea in pregnancy should not be attributed to pregnancy itself and a careful search for the cause of such signs and symptoms must be made so as to be able to find out the cause of these symptoms. Secondly the uncommon causes of fever should also be kept in differential diagnosis particularly when associated with features such as peculiar rash and appropriate diagnostic tests (in this case Weil-Felix test) must be advised so as to make an appropriate diagnosis. Anitharaj $\mathrm{V}$ et al, found that the sensitivity of Weil-Felix for diagnosis of scrub typhus has a sensitivity of more than $90 \%$ and reaching up to $99 \%{ }^{8}$

Since our patient had symptoms in immediate postpartum period an X-ray was feasible in antepartum period 2D echo (owing to its non-ionizing character) must be done if cardiac involvement is suspected. The other investigations which may help in the diagnosis of myocarditis is ECG which may show presence of paroxysmal atrial fibrillation. Chin JY et al in their study found that presence of ECG abnormalities such as ST elevation and PAT in patients of scrub typhus may be a sign of myocarditis. ${ }^{9}$

Management of scrub typhus in postpartum period depends upon the severity and may consist of antibiotics and supportive care. Cardiomyopathy needs to be treated in intensive care units and its management also depends upon severity. The treatment of cardiomyopathy is similar to any other case of myocarditis and may require administration of beta blockers, antiarrhythmics, ACE inhibitors and diuretics. ${ }^{10}$

\section{CONCLUSION}

Scrub typhus is one of the uncommon causes of febrile illness during peripartum period but still need to be kept in the differential diagnosis of cases presenting with fever, rash and signs and symptoms pointing towards ricketsial infection. Early diagnosis and proper treatment are key to management of patients with complications such as dilated cardiomyopathy in these patients.

Funding: No funding sources Conflict of interest: None declared

Ethical approval: Not required

\section{REFERENCES}

1. Karthik G, Sudarsan TI, Peter JV, Sudarsanam T, Varghese GM, Kundavaram P, et al. Spectrum of cardiac manifestations and its relationship to outcomes in patients admitted with scrub typhus infection. World J Crit Care Med. 2018;7(1):16-23.

2. Chipp E, Digby S. Rickettsia: an unusual cause of sepsis in the emergency department. Emerg Med J. 2006;23(11):e60.

3. Ayakawa $M$, Inoh $T$, Yokota $Y$, Kawanishi $H$, Matsumoto K, Kumaki T, Fukuzaki H. A long-term follow-up study of acute viral and idiopathic myocarditis. Jpn Circ J. 1983;47(11):1304-9.

4. Biesbroek PS, Beek AM, Germans T, Niessen HW, van Rossum AC. Diagnosis of myocarditis: Current state and future perspectives. Int $\mathrm{J}$ Cardiol. 2015;191:211-9. 
5. Rajan SJ, Sathyendra S, Mathuram AJ. Scrub typhus in pregnancy: Maternal and fetal outcomes. Obstet Med. 2016;9(4):164-6.

6. Rapsang AG, Bhattacharyya P. Scrub typhus. Indian J Anaesth. 2013;57(2):127-34.

7. Nieminen MS, Heikkilä J, Karjalainen J. Echocardiography in acute infectious myocarditis: relation to clinical and electrocardiographic findings. Am J Cardiol. 1984;53(9):1331-7.

8. Anitharaj V, Stephen S, Pradeep J, Park S, Kim SH, Kim YJ, Kim EY, et al. Serological diagnosis of acute scrub typhus in Southern India: Evaluation of InBios Scrub Typhus Detect IgM Rapid Test and comparison with other serological tests. J Clin Diagn Res. 2016;10(11):DC07-DC10.
9. Chin JY, Kang KW, Moon KM, Kim J, Choi YJ. Predictors of acute myocarditis in complicated scrub typhus: an endemic province in the Republic of Korea. Korean J Intern Med. 2018;33(2):323-30.

10. Maisch B, Alter P. Treatment options in myocarditis and inflammatory cardiomyopathy. Herz. 2018;43(5):423-30.

Cite this article as: Aggarwal A, Sharma MS. Unusual presentation of scrub typhus in pregnancy with postpartum dilated cardiomyopathy: a case report. Int J Reprod Contracept Obstet Gynecol 2020;9:1757-60. 